\title{
The diarrhetic shellfish-poisoning toxin, okadaic acid, provokes gastropathy, dysbiosis and susceptibility to bacterial infection in a non-rodent bioassay, Galleria mellonella
}

\author{
Helena Emery ${ }^{1} \cdot$ William Traves $^{1} \cdot$ Andrew F. Rowley $^{1} \cdot$ Christopher J. Coates $^{1}[$
}

Received: 6 July 2021 / Accepted: 4 August 2021 / Published online: 10 August 2021

(c) The Author(s) 2021

\begin{abstract}
Diarrhetic shellfish-poisoning (DSP) toxins such as okadaic acid and dinophysistoxins harm the human gastrointestinal tract, and therefore, their levels are regulated to an upper limit of $160 \mu \mathrm{g}$ per kg tissue to protect consumers. Rodents are used routinely for risk assessment and studies concerning mechanisms of toxicity, but there is a general move toward reducing and replacing vertebrates for these bioassays. We have adopted insect larvae of the wax moth Galleria mellonella as a surrogate toxicology model. We treated larvae with environmentally relevant doses of okadaic acid ( $80-400 \mu \mathrm{g} / \mathrm{kg}$ ) via intrahaemocoelic injection or gavage to determine marine toxin-related health decline: (1) whether pre-exposure to a sub-lethal dose of toxin $(80 \mu \mathrm{g} / \mathrm{kg}$ ) enhances susceptibility to bacterial infection, or (2) alters tissue pathology and bacterial community (microbiome) composition of the midgut. A sub-lethal dose of okadaic acid $(80 \mu \mathrm{g} / \mathrm{kg})$ followed $24 \mathrm{~h}$ later by bacterial inoculation $\left(2 \times 10^{5}\right.$ Escherichia coli) reduced larval survival levels to $47 \%$, when compared to toxin (90\%) or microbial challenge (73\%) alone. Histological analysis of the midgut depicted varying levels of tissue disruption, including nuclear aberrations associated with cell death (karyorrhexis, pyknosis), loss of organ architecture, and gross epithelial displacement into the lumen. Moreover, okadaic acid presence in the midgut coincided with a shift in the resident bacterial population over time in that substantial reductions in diversity (Shannon) and richness (Chao-1) indices were observed at $240 \mu \mathrm{g}$ toxin per kg. Okadaic acid-induced deterioration of the insect alimentary canal resembles those changes reported for rodent bioassays.
\end{abstract}

Keywords Histopathology $\cdot$ 16S (V3-V4) rRNA microbiome · Immune-compromised · In vivo model · Food poisoning · Marine toxins

\section{Introduction}

The lipophilic toxin okadaic acid and structurally similar dinophysistoxins are produced by several marine dinoflagellates, notably species of Prorocentrum and Dinophysis, and cause diarrhetic shellfish poisoning (DSP) in humans (Tachibana et al. 1981; Yasumoto et al. 1985; Trainer et al. 2013). DSP toxins are the most common output from harmful algal blooms in European, South American, and Asian waters, thereby representing the leading cause of aquaculture harvesting bans and site closures in Europe (Reguera et al.

Christopher J. Coates

c.j.coates@swansea.ac.uk

1 Department of Biosciences, Faculty of Science and Engineering, Swansea University, Swansea, Wales SA2 8PP, UK
2014; Dhanji-Rapkova et al. 2018; Bresnan et al. 2021). After consuming contaminated shellfish tissues, usually from phytoplankton filtering bivalves, humans can develop nausea, vomiting, incapacitating diarrhoea, abdominal cramps, and, in some cases, chills and fever within $0.5-5 \mathrm{~h}$. The structural integrity of DSP toxins remain intact after cooking and freeze-thawing (McCarron et al. 2008; Reboreda et al. 2010), and their presence in shellfish flesh does not appear to alter the organoleptic profile (Valdiglesias et al. 2013). Okadaic acid inhibits serine/threonine phosphatases (PP1, PP2A; Bialojan and Takai 1988), disrupting cellular homeostasis due to hyperphosphorylation and leading to downstream dysregulation of the gastrointestinal mucosa. The broad symptomology of okadaic acid-induced poisoning cannot be attributed to phosphatases alone and likely involves the enteric nervous system (e.g., neuropeptide Y; Louzao et al. 2015). Recent experiments carried out on Swiss female mice suggest that serotonin (5-hydroxytryptamine) levels are a 
contributing factor, as $0.1-10 \mathrm{mg} / \mathrm{kg}$ of cyproheptadine (a serotonin antagonist) delayed or prevented diarrhoea entirely (Louzao et al. 2021).

Beyond the GI tract, organs such as the kidneys, liver, and placenta can be affected, with okadaic acid demonstrating mutagenic, genotoxic, carcinogenic, and neurotoxic properties (Matias and Creppy 1996; reviewed by Mundy 2013). Chronic exposure to this polyether toxin is linked to elevated levels of tumour promoters and increased risk of cancers, e.g., colorectal (Manerio et al. 2008; Vilariño et al. 2018; Jiménez-Cárcamo et al. 2020). Because of the diverse biological properties, distribution, and frequency of DSP toxins in the marine environment, their levels are regulated across Europe-with an upper limit of $160 \mu \mathrm{g}$ [toxin equivalents] per kg [shellfish tissue] - to protect consumers (O’Mahony 2018). The acute, subacute and chronic, low-dose immunotoxicological effects of shellfish-poisoning toxins (including DSPs) are poorly defined and understudied in humans, as are the detrimental impact they have across marine food webs, including commercial finfish and shellfish (Coates and Söderhäll 2020; Corriere et al. 2020, reviewed by Turner et al. 2021).

For many years, small vertebrates (e.g., rodents, zebrafish) and invertebrates (e.g., fruit flies, nematodes) have been used as models/proxies for humans across the biological and biomedical sciences including the study of genetics, senescence, host-pathogen interactions, and antibiotic development. Regarding invertebrates, their use brings obvious financial, time, and ethical advantages, however, those organisms mentioned above are rather small-making precise dosing challenging-despite their high-throughput capacity (Emery et al. 2019, 2021). Larvae of the greater wax moth (Galleria mellonella) offer combined benefits: low cost of maintenance, accurate dose administration via several routes (topical, intrahaemocoelic injection, gavage), can be used at human relevant $37^{\circ} \mathrm{C}$, and key cellular immune functions (e.g., pathogen recognition, internalisation, and destruction) mirror aspects of vertebrate innate immunity (Browne et al. 2013; Lim et al. 2018; Lange et al. 2018, 2019). For these reasons, the popularity of G. mellonella continues to increase as an experimental alternative for screening microbial virulence and pathogenicity, efficacy of antimicrobials, biocontrol agents, and to discriminate between toxic and non-toxic substances (Alghoribi et al. 2014; Champion et al. 2016; Allegra et al. 2018; de Barros et al. 2019; Grizanova et al. 2019, 2021; Piatek et al. 2020; Emery et al. 2021; Moya-Andérico et al. 2021; Krachler et al. 2021). Recently, we reported on the effects of G. mellonella exposure to environmentally relevant doses of okadaic acid $(\sim 80-400 \mu \mathrm{g} / \mathrm{kg})$ and recorded cytotoxic (immune cell death) and gastrotoxic (REDOX imbalance) effects similar to known toxicological endpoints for the established mouse bioassay (Coates et al. 2019). This study demonstrated the potential use of G. mellonella for assessing the risk of marine toxins in vivo, or complementing in vitro cell-line-based assays (e.g., Caco-2) prior to vertebrate use.

In the present study, our overall aim was to define okadaic acid-related health decline and pathophysiological condition in the aspirant toxicology model, G. mellonella. First, we determined whether pre-exposure of larvae to a low dose of okadaic acid $(80 \mu \mathrm{g} / \mathrm{kg})$, followed $24 \mathrm{~h}$ later by $2 \times 10^{5}$ CFUs of a common laboratory bacterium (Escherichia coli), led to enhanced susceptibility to infection. Second, we used tissue histology and 16S rRNA high-throughput sequencing (V3-V4 region) to compare pathological modalities between the intoxicated (mid)gut tissues of G. mellonella and rodent bioassays.

\section{Materials and methods}

\section{Toxin and bacterium}

Okadaic acid $\left(\mathrm{C}_{44} \mathrm{H}_{68} \mathrm{O}_{13}\right)$ was purchased from TOCRIS Biosciences (UK; Cat. No. 1136). Solutions of okadaic acid were prepared in $0.2 \mu \mathrm{m}$ filter-sterilised phosphate-buffered saline (PBS) pH 7.4 containing 5\% dimethyl sulfoxide (DMSO; v/v). All remaining reagents, unless stated otherwise, were sourced from MERCK (formerly Sigma-Aldrich, Dorset, UK) in their purest form available. The bacterial target Escherichia coli K12 (LZB 035) was sourced from Blades Biological Ltd (Kent, UK) on nutrient agar slopes.

\section{Galleria mellonella intoxication and infection trials}

Final instar larvae (G. mellonella) were purchased from Live Foods Direct Ltd (Sheffield, UK) and stored at $14-15^{\circ} \mathrm{C}$ in the dark for no more than 5 days prior to use. Healthy larvae weighing 250-320 mg each-showing no signs of pupation, melanisation, or infection - were administered with okadaic acid either by intrahaemocoelic injection (INJ) through the last left (ventral) pro-leg or orally (force-fed, FF) using a sterile (disposable) 27-gauge hypodermic needle across the concentration range $80-400 \mu \mathrm{g} / \mathrm{kg}$. Larvae were chilled on ice for no more than $2 \mathrm{~min}$ immediately prior to inoculation. The negative control consisted of PBS pH 7.4 containing $5 \%(\mathrm{v} / \mathrm{v})$ DMSO. The volume was standardised to $10 \mu \mathrm{l} \mathrm{per}$ insect regardless of the inoculation route (gavage/injection) or the contents of the inoculum (chemical/microbial). Postintoxication, larvae were incubated in the dark at $30^{\circ} \mathrm{C}$. The health of each larva was assessed over a 72-h period using an abridged scoring system developed by Loh et al. (2013) for this insect species [melanisation (0-4), activity (0-3)]. Levels of melanisation are used as indicators of infection/ immunity/stress in insects (Whitten and Coates 2017) — detrimental accumulation of the black/brown pigment scores 0 
(i.e., highly melanised, compromised larvae), whereas an integument free of pigmentation scores 4 (i.e., healthy, naive larvae).

Single colonies of bacterial targets, E. coli (Gram-negative), were selected from nutrient agar and sub-cultured into nutrient broth and grown overnight at $30{ }^{\circ} \mathrm{C}(200 \mathrm{rpm})$. Bacteria were handled using standard axenic practices. Once bacterial suspensions reached an optical density $\left(\mathrm{OD}_{600}\right)$ value of 1 (VWR 1200 spectrophotometer), cells were centrifuged at $1000 \times \mathrm{g}$ for $5 \mathrm{~min}$, washed once in PBS pH7.4, and then diluted to the required doses. The numbers (dose) of bacterial colony-forming units (CFUs) used in infection studies were $2 \times 10^{5}, 5 \times 10^{5}, 1 \times 10^{6}$, and $2 \times 10^{6}$ per insect.

In the final set of toxicity studies, insects were preexposed to the lowest dose of okadaic acid via force feeding $(80 \mu \mathrm{g} / \mathrm{kg})$, followed $24 \mathrm{~h}$ later by $E$. coli $\left(2 \times 10^{5}\right)$ challenge directly into the haemocoel. Controls consisted of the following inoculation combinations, PBS (FF) + PBS (INJ), PBS (FF) + bacteria (INJ), and OA (FF) + PBS (INJ). Larvae were surface sterilised with $70 \%$ ethanol prior to any microbial inoculation to avoid potential contamination from the integument.

\section{Tissue histology of larval midgut}

Larvae were inoculated orally with OA (80 and $240 \mu \mathrm{g}$ / $\mathrm{kg}$ ) or PBS (+5\% DMSO)—doses determined previously by Coates et al. (2019) — and sacrificed at 4, 24, and $48 \mathrm{~h}$ by fixation in $10 \%$ formalin overnight at room temperature. Fixed larvae were cut into three parts; head, middle, and rear in preparation for paraffin wax embedding. Samples were dehydrated in $70 \%, 80 \%$, and $90 \%$ ethanol each for $1 \mathrm{~h}$ followed by three 1-h $100 \%$ ethanol washes. Dehydrated samples were washed in Histoclear twice for $1 \mathrm{~h}$ to remove any remaining fixative, followed by $50 \%$ Histoclear: $50 \%$ paraffin wax for $1 \mathrm{~h}$. Wax blocks containing insect tissues were trimmed manually to minimise cutting and staining areas. Trimmed blocks were sliced into 5-7 $\mu \mathrm{m}$ sections using a microtome, loaded onto glass slides with water, and dried on a heating block to allow the wax ribbon to fully expand and stick to the surface of the glass slide. Slides were staind with haematoxylin and eosin as described by Emery et al. (2019).

\section{Gut microbiome analysis of okadaic acid-intoxicated larvae}

Randomly selected larvae ( $n=3$ per treatment, per time point, $n=27$ overall) that were force-fed okadaic acid ( 80 or $240 \mu \mathrm{g} /$ larva) or untreated $(0 \mu \mathrm{g} /$ larva) were chilled on ice and dissected at 4, 24, and $48 \mathrm{~h}$. Gut tissues (mouth to anus) were removed carefully from each larva, weighed, snap frozen in liquid nitrogen, and stored at $-80{ }^{\circ} \mathrm{C}$ before genomic DNA extraction using a Qiagen DNeasy Blood and Tissue Kit (Cat. No. 69504; Qiagen, Hilden, Germany) by following the manufacturer's recommended protocol. DNA yields were quantified using the Qubit ${ }^{\mathrm{TM}}$ dsDNA High Sensitivity Assay Kit and Qubit ${ }^{\mathrm{TM}}$ Fluorometer (Invitrogen, California, USA) and standardised to $50 \pm 2 \mathrm{ng} / \mu \mathrm{l}$ prior to sequencing.

Eurofins Genomics (Germany) carried out the highthroughput sequencing using their established INVIEW Microbiome Profiling 3.0 service - performed on an Illumina MiSeq platform targeting the V3-V4 hypervariable $16 \mathrm{~S}$ ribosomal RNA region $(2 \times 250-300 \mathrm{bp})$ with the following oligonucleotide primers: Forward: TACGGG AGGCAGCAG (Turner et al. 1999) Reverse: CCAGGG TATCTAATCC (Kisand et al. 2002). Amplicon generation, adapter addition, quality control, size selection, pooling, demultiplexing, removal of primers, and advanced bioinformatics were performed by Eurofins (raw data processing, read merging, quality filtering, and chimera removal). De-multiplexing was carried out on all reads that were approved by the standard Illumina chastity filter. Read merging was completed using the FLASH algorithm to create a consensus sequence with the greatest quality value, considered all overlaps, and produced merged readings with the longest targeted region (Magoč and Salzberg, 2011). Where merging was not possible, the forward read was used, and merged readings were then filtered, removing reads that were too long/short. Initial microbiome profiling to remove chimeric reads was conducted using UCHIME followed by entropy decomposition analysis to form datasets of partition marker genes within operational taxonomic units (OTUs; Schloss et al. 2011; Eren et al. 2015). Each OTU was assigned using a minimum of $70 \%$ sequence identity across a minimum representative sequence of $80 \%$ using DC-Megablast (Altschul et al. 1990). OTUs were processed using QIIME software v1.9.1 (Caporaso et al. 2010) with a 97\% homogeneity threshold for selection. OTUs lacking taxonomic matches were categorised as 'unclassified' and taxonomic units with $<0.1 \%$ reads were considered 'other'.

OTUs at phylum and genus levels were analysed in $\mathrm{R}$ studio using the microbiome and vegan packages to calculate Chao-1 (richness) and Shannon (diversity) indices, and the randomised permutations test; adonis PERMANOVA (Bray-Curtis method with 999 permutations) was conducted to assess signficance between microbiomes (Cree et al. 2016). Stress and dispersion tests were performed and plotted to ensure that the model was a good fit. All samples were filtered to remove putative contaminants using any reads present in the extraction blank-OTU reads $\leq 200$ were removed, leaving the remaining abundances for analysis. Replicates were merged to create average OTU values for each treatment/time point. A single sample, untreated larva at $24 \mathrm{~h}$ did not yield any sequence data. 


\section{Data handling and statistical analyses}

Experiments were repeated on at least three separate occasions. Results are expressed as mean \pm SE (unless stated otherwise), and sample sizes can be found within the respective figure descriptors. Log-rank (Mantel-Cox) tests were used to analyse survival curves, whereas two-way ANOVAs (with Turkey's multiple comparison tests) were used for health/damage indices in GraphPad PRIMS v7. The D'Agostino-Pearson test (Omnibus K2) was used to check data for normality, and $\log$-transformed $[\log (y+1)]$ when necessary. Significance values were determined when $P \leq 0.05$. Microbiome analysis (16S region) was performed in $\mathrm{R}$ studio as described above.

Histology slides were visualised and singled-blind assessed as oulined in Emery et al. (2019). Briefly, a scoring index from 1 to 4 was used to indicate visible damage: (1) little change (if any), 0-2 localised tissue aberrations per slide, (2) discrete changes of 3 to 5 tissue aberrations per slide, (3) regional change representing $\geq 25 \%$ damage (the alteration is dramatic), and (4) global change of $>50 \%$ of a specific tissue type or the entire slide (gross). Images of tissue sections were adjusted for colour balance and contrast/ brightness.

\section{Results}

\section{Susceptibility of Galleria mellonella to bacteriosis in the absence and presence of okadaic acid}

Administering single doses of either okadaic acid via gavage (Fig. 1) or E. coli via intrahaemocoelic injection (Fig. 2) led to significant declines in larval health in a dose-dependent manner $\left(X_{(4)}^{2}=110.7, P<0.0001\right.$ [Fig. 1a] and $X^{2}{ }_{(5)}=157.9$, $P<0.0001$ (Fig. 2a); Table 1). Larval survival levels
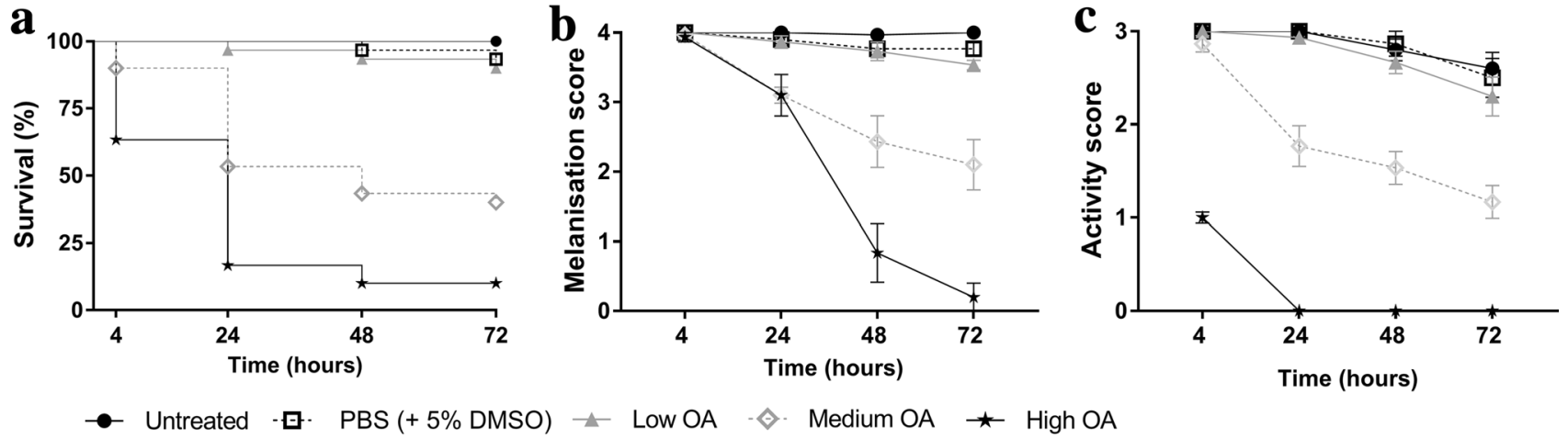

Fig. 1 Health indices of Galleria mellonella force-fed okadaic acid $\left(\mathrm{C}_{44} \mathrm{H}_{68} \mathrm{O}_{13}\right)$. Insects were exposed to $80 \mu \mathrm{g}$ (low), $240 \mu \mathrm{g}$ (medium), or $400 \mu \mathrm{g}$ (high) okadaic acid and monitored for survival (a), melanisation (b), and activity (c) levels across the 72-h experimental period. Post-inoculation, larvae were incubated at $30{ }^{\circ} \mathrm{C}$ in the dark. Untreated larvae received no injection, and the negative control consisted of PBS $(+5 \%[\mathrm{v} / \mathrm{v}]$ DMSO). Values represent the mean \pm S.E. $(n=30$ per treatment, 150 in total)

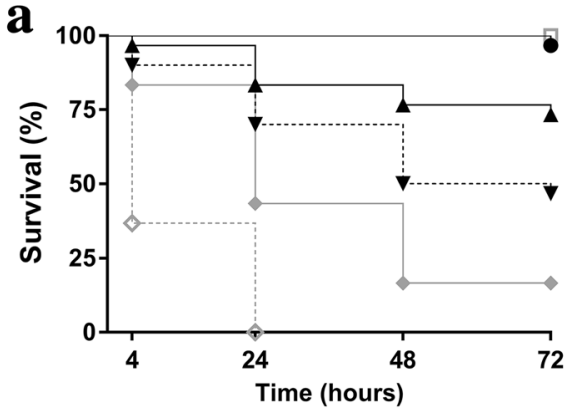

- Unchallenged - ㅁ. PBS (+5\% DMSO)
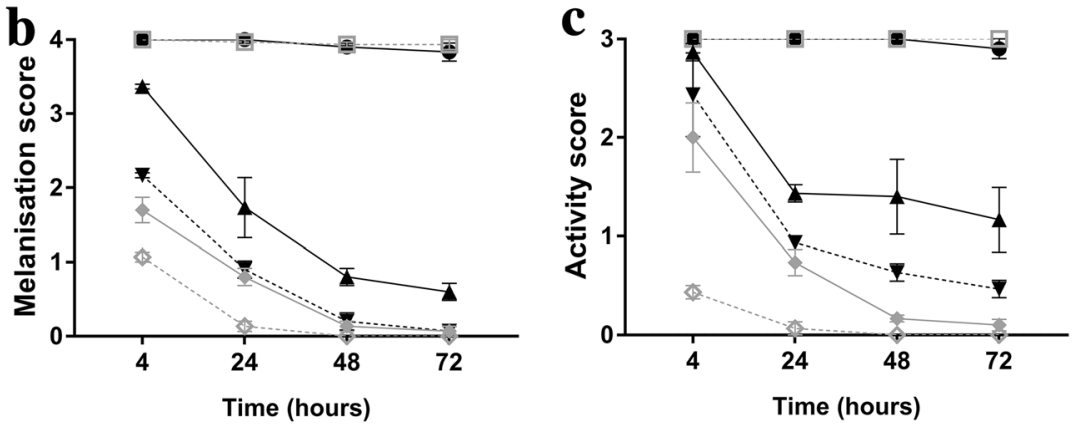

$-2 \times 10^{5}$

$5 \times 10^{5} \diamond 1 \times 10^{6} \diamond 5 \times 10^{6}$
Fig. 2 Health indices of Galleria mellonella infected with bacteria via intrahaemocoelic injection. Insects were exposed to Escherichia coli across the range, $2 \times 10^{5}-5 \times 10^{6} \mathrm{CFUs}$, and monitored for survival (a), melanisation (b), and activity (c) levels during the 72-h experimental period. Post-inoculation, larvae were incubated at $30{ }^{\circ} \mathrm{C}$ in the dark. Untreated larvae received no injection, and the negative control consisted of PBS $(+5 \%[\mathrm{v} / \mathrm{v}]$ DMSO). Values represent the mean \pm S.E. $(n=30$ per treatment, 180 in total $)$ 
Table 1 Two-way analysis of variance (ANOVA) for Galleria mellonella health indices

\begin{tabular}{|c|c|c|c|c|c|c|}
\hline & Source of variation & Variation (\%) & Sum-of-squares & $d f$ & $F$ value & $P$ value \\
\hline \multicolumn{7}{|l|}{ Intoxication (OA) } \\
\hline \multirow[t]{3}{*}{ Melanisation index } & Time & 17.5 & 0.314 & 3 & 27.3 & $<0.0001$ \\
\hline & Treatment & 42 & 0.753 & 4 & 49.2 & $<0.0001$ \\
\hline & Interaction & 31.9 & 0.571 & 12 & 12.5 & $<0.0001$ \\
\hline \multirow[t]{3}{*}{ Activity index } & Time & 6.8 & 0.182 & 3 & 66.5 & $<0.0001$ \\
\hline & Treatment & 86 & 2.289 & 4 & 628.6 & $<0.0001$ \\
\hline & Interaction & 5.7 & 0.151 & 12 & 13.8 & $<0.0001$ \\
\hline \multicolumn{7}{|l|}{ Infection (E. coli) } \\
\hline \multirow[t]{3}{*}{ Melanisation index } & Time & 15.6 & 0.854 & 3 & 167.8 & $<0.0001$ \\
\hline & Treatment & 74.8 & 4.1 & 5 & 483.9 & $<0.0001$ \\
\hline & Interaction & 8 & 0.446 & 15 & 17.6 & $<0.0001$ \\
\hline \multirow[t]{3}{*}{ Activity index } & Time & 11.8 & 0.452 & 3 & 60.8 & $<0.0001$ \\
\hline & Treatment & 77 & 2.96 & 5 & 238.5 & $<0.0001$ \\
\hline & Interaction & 8.1 & 0.31 & 15 & 8.3 & $<0.0001$ \\
\hline \multicolumn{7}{|c|}{ Intoxication and infection } \\
\hline \multirow[t]{3}{*}{ Melanisation index } & Time & 21.2 & 0.439 & 3 & 76.1 & $<0.0001$ \\
\hline & Treatment & 47.9 & 0.994 & 4 & 129 & $<0.0001$ \\
\hline & Interaction & 27.2 & 0.563 & 12 & 24.4 & $<0.0001$ \\
\hline \multirow[t]{3}{*}{ Activity index } & Time & 22.6 & 0.151 & 3 & 50.22 & $<0.0001$ \\
\hline & Treatment & 40.6 & 0.270 & 4 & 67.6 & $<0.0001$ \\
\hline & Interaction & 30.8 & 0.21 & 12 & 17.1 & $<0.0001$ \\
\hline
\end{tabular}

$d f$ degrees of freedom; $O A$ okadaic acid $(80 \mu \mathrm{g} / \mathrm{kg}) ;$ Escherichia coli $\left(2 \times 10^{5}\right.$ per insect $)$ dropped by $10 \%$ across the 72 -h experimental period when $80 \mu \mathrm{g} / \mathrm{kg}$ of okadaic acid was used, which is half the upper regulatory limit (i.e., $160 \mu \mathrm{g} / \mathrm{kg}$ ) set by the European Union (EC No. 853/2004). At doses of 240 and $400 \mu \mathrm{g} / \mathrm{kg}$, survival levels reduced substantially by $60 \%$ and $90 \%$, respectively (Fig. 1a; Supplementary Table 1). At the two higher doses, there was concomitant reductions in melanisation $(<2.1$ out of 4; Fig. 1b) and activity ( $<1.2$ out of 3; Fig. 1c) indices for the intoxicated insects. Similarly, increasing the number of E. coli colony-forming units (CFUs) from $2 \times 10^{5}$ to $5 \times 10^{6}$ led to reciprocal deteriorations in melanisation $(<0.6$ out of 4; Fig. 2b) and activity ( $<1.2$ out of 3; Fig. 2c) indices at 72 -h post-infection. In fact, no larvae survived beyond $24 \mathrm{~h}$ when infected with $5 \times 10^{6} \mathrm{CFUs}$ E. coli. For okadaic acid-related health decline, time and treatment accounted for $\sim 7-18 \%$ and $\sim 42-86 \%$ of the variation within the data, respectively. For $E$. coli-related health decline, time and treatment accounted for $\sim 12-16 \%$ and $~ 76 \%$ of the variation within the data, respectively. In all cases, time, treatment, and their interaction were significant contributing factors $(P<0.0001$; Table 1$)$ to larval morbidity.

Pre-exposing $G$. mellonella to a low dose of okadaic acid $(80 \mu \mathrm{g} / \mathrm{kg})$, followed $24 \mathrm{~h}$ by $2 \times 10^{5}$ CFUs of $E$. coli, caused survival levels to drop by $>53 \%$ within $48 \mathrm{~h}$ (Fig. 3a; $\left.X^{2}{ }_{(4)}=42.23, P<0.0001\right)$. Changes also corresponded with the lowest melanisation and activity indices recorded, $<0.3$ out of 4 and $\sim 1$ out of 3 , respectively (Table 1; Fig. 3b, c). Moreover, calculated hazard ratios suggest that insects receiving both toxin and bacterium were 2.7 - to 6.3 -fold more likely for death to occur over the same time period when compared to those receiving either challenge alone (Table 2). By replacing okadaic acid (first inoculum) or $E$. coli (second inoculum) with PBS, significantly fewer larval deaths were encountered (Table 2 for statistical outputs), with survival levels remaining above $73 \%$ (Fig. 3a). Using two inoculation routes, i.e., oral and intrahaemocoelic injection per larva, did not appear to compromise health, as indices were similar to the untreated group $(P=0.317$; Table 2; Fig. 3a-c).

\section{Effect of okadaic acid on the gut tissues of Galleria mellonella}

a. Histopathology

Histology slides were prepared from insects force-fed PBS or okadaic acid, and single blind assessed using the same grading system from Emery et al. (2019). Midgut tissues examined from untreated and negative controls (PBS $+5 \%$ DMSO) showed some variation in architecture but contained few clear signs of damage; all slides were assigned grade 1 ( $<2$ discrete changes), apart from two PBS slides graded 2 (one at 4 and another at 48 hours; Fig. 4). Transverse and longitudinal sections along the midgut of PBS-treated larvae revealed an 

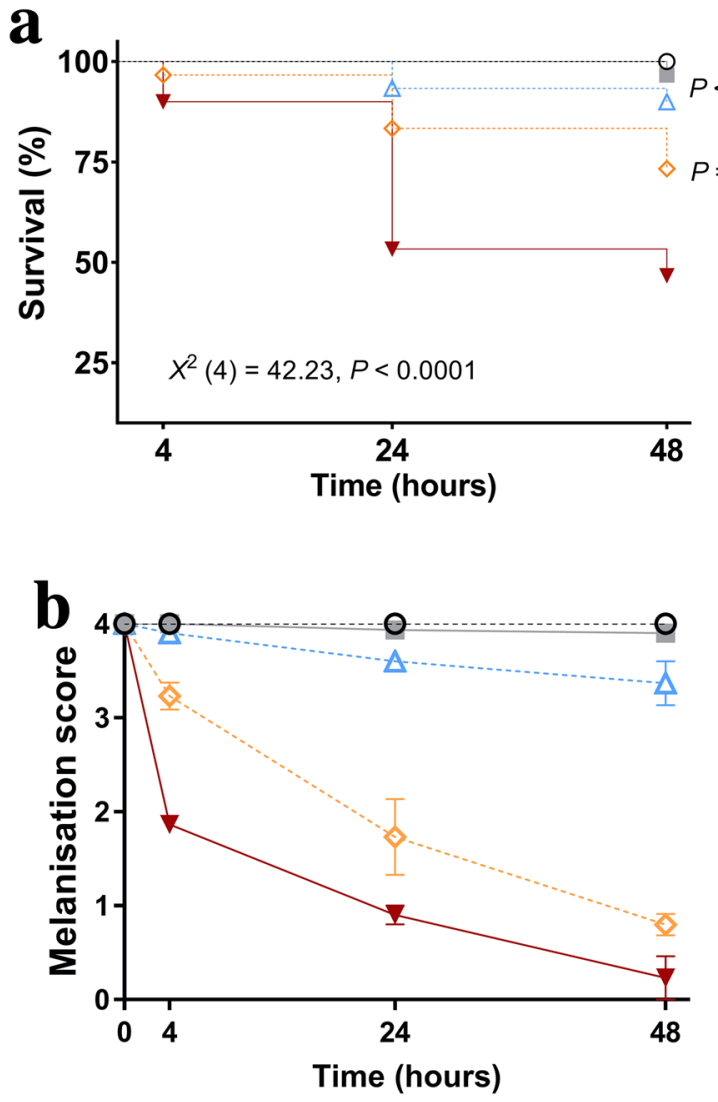

Fig. 3 Health indices of Galleria mellonella challenged with a combination of okadaic acid and bacteria. Insects were pre-exposed to the low dose of okadaic acid $(80 \mu \mathrm{g} / \mathrm{kg})$ via gavage, followed $24 \mathrm{~h}$ later by an intrahaemocoelic injection of E. coli $\left(2 \times 10^{5}\right.$ CFUs). After bacterial inoculation, survival (a), melanisation (b), and activity (c) levels were monitored for a further $48 \mathrm{~h}$. Larvae were incubated at $30^{\circ} \mathrm{C}$ in the dark. Untreated larvae received no injection, and the negative
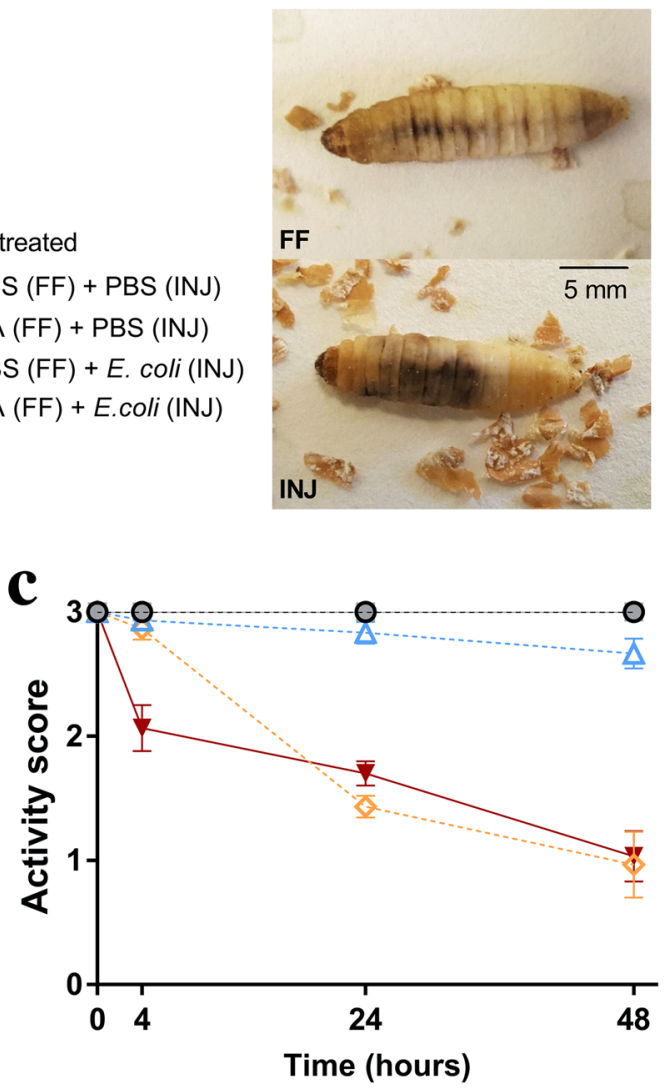

control consisted of PBS (+5\% [v/v] DMSO) administered first by gavage, followed $24 \mathrm{~h}$ later by an injection into the haemocoel. Values represent the mean \pm S.E. $(n=30$ per treatment, 150 in total). Inset, images of melanisation $24 \mathrm{~h}$ post-force feeding (FF) an insect larva okadaic acid, or injecting bacteria directly (INJ) into the body cavity. Darkening is restricted to the alimentary canal (laterally from mouth to anus) when insects are treated orally

Table 2 Survival analyses of Galleria mellonella larvae inoculated with okadaic acid and bacteria alone, or in succession

\begin{tabular}{|c|c|c|c|c|}
\hline & PBS (FF) + PBS (INJ) & $\mathrm{OA}(\mathrm{FF})+\mathrm{PBS}(\mathrm{INJ})$ & $\mathrm{PBS}(\mathrm{FF})+E . \operatorname{coli}(\mathrm{INJ})$ & $\mathrm{OA}(\mathrm{FF})+$ E. coli $(\mathrm{INJ})$ \\
\hline Untreated & \multicolumn{2}{|c|}{$X^{2}(1)=1, P=0.3173 X^{2}(1)=3.106, P=0.078$} & $X^{2}(1)=9.085 \boldsymbol{P}=\mathbf{0 . 0 0 5 2 6}$ & $X^{2}(1)=21.59, P<0.0001$ \\
\hline $\mathrm{PBS}(\mathrm{FF})+\mathrm{PBS}(\mathrm{INJ})$ & - & $\begin{array}{l}X^{2}(1)=1.09, P=0.296 \\
\mathrm{HR}=2.87(95 \% \mathrm{CI} \\
0.39-20.72)\end{array}$ & $\begin{array}{l}X^{2}(1)=6.468, \boldsymbol{P}=\mathbf{0 . 0 1 1 0} \\
\mathrm{HR}=5.7(95 \% \mathrm{CI}, 1.49- \\
21.81)\end{array}$ & $\begin{array}{l}X^{2}(1)=18.74, \boldsymbol{P}<\mathbf{0 . 0 0 0 1} \\
\mathrm{HR}=9.71(95 \% \text { CI, 3.5-26.69) }\end{array}$ \\
\hline $\mathrm{OA}(\mathrm{FF})+\mathrm{PBS}(\mathrm{INJ})$ & - & - & $\begin{array}{l}X^{2}(1)=2.77, P=0.95 \\
\mathrm{HR}=2.83(95 \% \text { CI, 0.83-9.6) }\end{array}$ & $\begin{array}{l}X^{2}(1)=13.37, \boldsymbol{P}=\mathbf{0 . 0 0 0 3} \\
\mathrm{HR}=6.32(95 \% \text { CI, } 2.54-15.4)\end{array}$ \\
\hline $\mathrm{PBS}(\mathrm{FF})+$ E. coli $(\mathrm{INJ})$ & - & - & - & $\begin{array}{l}X^{2}(1)=4.821, \boldsymbol{P}=\mathbf{0 . 0 2 8 1} \\
\mathrm{HR}=2.72(95 \% \text { CI }, 1.1-6.65)\end{array}$ \\
\hline
\end{tabular}

Values represent pairwise comparisons between curves using the log-rank (Mantel-Cox) test and Mantel-Haenszel hazard ratios (HR). Significant differences were confirmed when $P<0.05$, and are highlighted in bold. Okadaic acid $(O A, 80 \mu \mathrm{g} / \mathrm{kg})$ was administered via force feeding $(F F)$. Escherichia coli $\left(2 \times 10^{5} \mathrm{CFUs}\right)$ was administered via intrahaemocoelic injection (INJ)

intact arrangement of epithelial cells, including columnar, goblet, and regenerative cells (Fig. 5A-H). Epithelial folds, involved in nutrient absorption, were identi- fied easily (Fig. 5B, D), as were the brush borders (with peritrophic matrix), basement membrane, and underlying muscle layer (Fig. 5C, G, H). The midgut lumen 


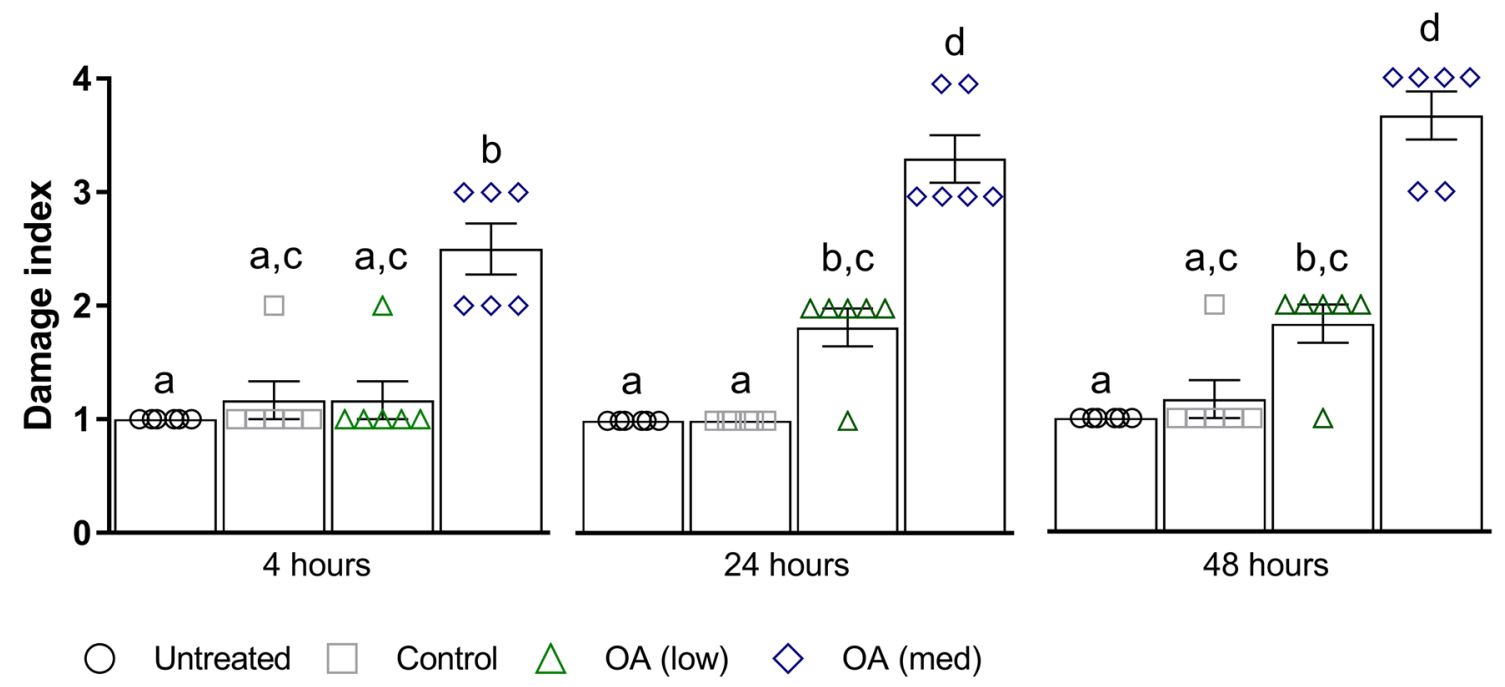

Fig. 4 Extent of midgut tissue damage in Galleria mellonella after feeding okadaic acid. Okadaic acid doses represent $80 \mu \mathrm{g} / \mathrm{kg}$ (low) and $240 \mu \mathrm{g} / \mathrm{kg}$ (high). Control, or PBS + 5\% (v/v) DMSO. Histology slides were single blind assessed in pairs (treatment vs control) and subsequently assigned a grade (1-4) based on damage. Grade 1 indicates little-to-no damage, whereas grade 4 represents global damage

appeared mostly free of cellular debris (Fig. 5A, D, E), and there were few signs of immune cell (haemocyte) presence within the body cavity immediately surrounding the midgut (Fig. 5C).

The low dose of OA $(80 \mu \mathrm{g} / \mathrm{kg})$ inflicted discrete/ localised injury compared to the untreated and negative controls, which was apparent after $24-48 \mathrm{~h}$ (Fig. 4). The higher dose of OA $(240 \mu \mathrm{g} / \mathrm{kg})$ caused obvious damage to the larval midgut within the first $4 \mathrm{~h}$ of force feeding and became more extensive over the proceeding $48 \mathrm{~h}$ (Figs. 4, 6). Okadaic acid intoxication manifested as displacement of cells into the gut lumen, membrane blebbing/blistering, loss of cellular morphology, vacuolisation, and nuclear aberrations (pyknosis, karyolysis, and karyorrhexis) associated with cell death. Entire regions sloughed into the lumen by 4 and $24 \mathrm{~h}$ (Fig. 6A-D). In the body cavity, haemocytes were damaged and fragmented (Fig. 6E), which indicated the toxin made its way across the protective sublayers of the midgut. By $48 \mathrm{~h}$, global tissue damage was apparent, and in extremis, the integrity of the entire midgut was compromised by necrosis and gross melanisation (Fig. 6G, H). Overall, okadaic acid jeopardised the insect alimentary canal with treatment (i.e., dose) and time accounting for $79 \%$ and $<4 \%$, respectively, of the variation within the data (Treatment, $\mathrm{F}_{(3,60)}=124.2, P<0.0001$; Time, $\mathrm{F}_{(2,60)}=8.2, P=0.0007$; Fig. 4).

b. Resident bacteria (microbiome)

Microbial signals in genomic DNA extracts of G. mellonella were interrogated by amplifying $16 \mathrm{~S}$ (V3-V4) affecting $>50 \%$ of tissue. Data (mean $+/-$ S.E.) have been compiled from assessments carried out at 4,24 , and $48 \mathrm{~h}$ post-inoculation. Unshared letters indicate significant differences $(P<0.05)$ determined by ANOVA and Tukey's multiple comparison tests ( $n=3$ insects (and two technical replicates) per category per time point, 36 in total)

rRNA and sequencing using Illumina MiSeq. General diversity among the bacterial taxa colonising the gut tissues was low, whether intoxicated or naïve. In the absence of toxin, bacterial operational taxonomic units (OTUs) for the gut were dominated by four phyla in ascending order (Fig. 7): Actinobacteria (5-9\%), Bacteroidetes (8-24\%), Proteobacteria (20-23\%), and Firmicutes (45-59\%). Such abundance and composition are in good agreement with the previous studies (e.g., Dubovskiy et al. 2016). Oral administration of $80 \mu \mathrm{g} / \mathrm{kg}$ okadaic acid coincided with a transient shift in bacterial composition at $4 \mathrm{~h}$ post-exposure to $\sim 90 \%$ Firmicutes and $<1 \%$ Actinobacteria but seemed to return to (and exceed) pre-toxin diversity levels after 24-48 h. Conversely, using the higher dose of $240 \mu \mathrm{g} / \mathrm{kg}$ led to obvious reductions in all representative phyla (Acidobacteria and Spirochaetes were undetectable), except for Firmicutes, and corresponded to decreased Shannon (alpha) diversity and Chao- 1 richness indices $(3.7 \pm 0.2$ and $333 \pm 21$, respectively) compared to those fed $80 \mu \mathrm{g} / \mathrm{kg}$ $($ Shannon $=4.2 \pm 0.34$; Chao- $1=438 \pm 82)$ and $0 \mu \mathrm{g} /$ $\mathrm{kg}($ Shannon $=4.5 \pm 0.1$; Chao- $1=453 \pm 17)$ when considering all time points (Fig. 7). When probing down to genus level, Enterococcus represented $~ 46-67 \%$ (0 $\mu \mathrm{g} / \mathrm{kg}$ toxin), $\sim 30-91 \%$ (80 $\mu \mathrm{g} / \mathrm{kg}$ toxin), and $91-98 \%$ ( $240 \mu \mathrm{g} / \mathrm{kg}$ toxin) of the top 35 identifiable taxa (Fig. 8), which does complement previous observations of enterococci prevalence across diverse G. mellonella tissue types (e.g., skin, gut, fat body, haemolymph; Krams et al. 2017; Allonsius et al. 2019). Interestingly, after 

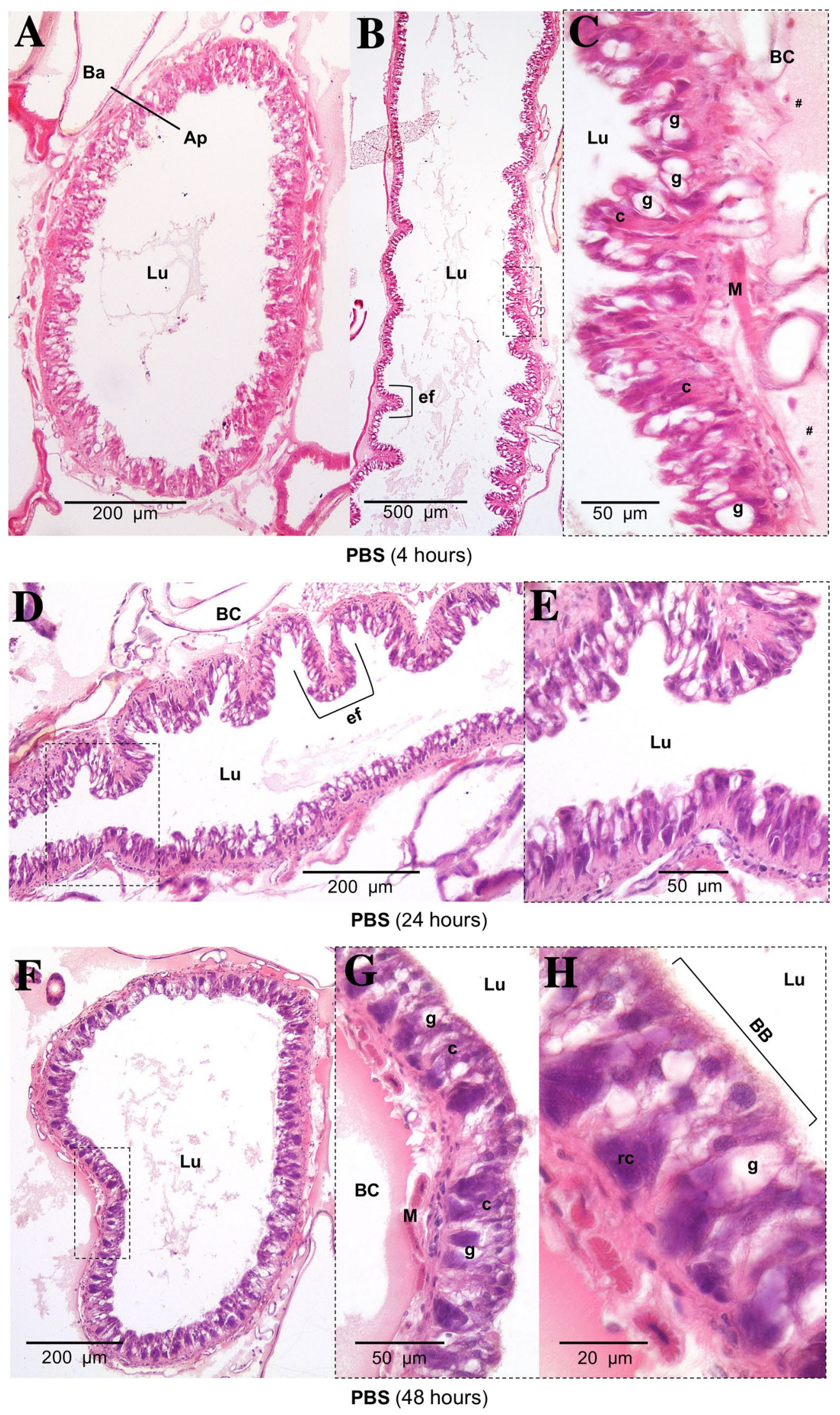
४Fig. 5 Tissue sections of the Galleria mellonella midgut when forcefed PBS. Photomicrographs depict transverse and longitudinal sections at 4 (panels A, B, C), 24 (panels D, E), and 48 (panels F, G, $\mathrm{H})$ h post-inoculation. $A p$ apical; $B a$ basolateral; $B B$ brush border; $B C$ body cavity; $c$ columnar (epithelial) cell; $e f$, epithelia fold(s); $g$, goblet cell; $L u$, lumen; $M$, muscle; $r c$, regeneration cell. Hashtags (\#) denote haemocytes

48-h exposure to a low dose of okadaic acid $(80 \mu \mathrm{g} / \mathrm{kg})$, larvae displayed $\sim 30 \%$ each Enterococcus and Lactobacillus - perhaps the population was disrupted to such an extent that minor taxa could gain a foothold (both genera represent the Firmicutes).

Group-wise (community-level) comparisons on bacterial composition were analysed using PERMANOVA (999 permutations based on the Bray-Curtis method). Overall, the higher toxin dose $(240 \mu \mathrm{g} / \mathrm{kg})$ had a significant effect on the microbial composition when compared to the control larvae $\left(0 \mu \mathrm{g} / \mathrm{kg} ; P=0.003, R^{2}=0.172\right)$, however, was non-significant when compared to the lower dose $\left(80 \mu \mathrm{g} / \mathrm{kg} ; P=0.3, R^{2}=0.0911\right.$; Supplementary Table 2; Supplementary Fig. 1).

\section{Discussion}

We assessed the putative impact of okadaic acid-one of the most common marine toxins and shellfish contaminants globally —on the integrity of gastric tissues and their resident microbiota using an alternative bioassay (G. mellonella larvae). In doing so, we accrued clear evidence that okadaic acid exposure at half $(80 \mu \mathrm{g} / \mathrm{kg})$ the upper regulated limit of $160 \mu \mathrm{g} / \mathrm{kg}$ enhanced susceptibility to infection by $>15 \%$ (Fig. 3a), and this acute event, coincided with visible damage to the gut epithelium and transient reductions in bacterial taxonomic diversity and richness (Figs. 4, 7). Increasing the acute dose to $240 \mu \mathrm{g} / \mathrm{kg}$ - approaching the published $\mathrm{LD}_{50}$ values for rodents (e.g., Aune et al. 2007, 2012) — caused extensive tissue disintegration, alongside prolonged bacterial dysbiosis (Figs. 6,7). The severity of tissue damage was such that haemocytes (immune cells) circulating in the haemocoel (body cavity) surrounding the midgut were impaired (Fig. 6E) —indicating the toxin leaked across the no-longer-intact epithelium and underlying muscle. Immune-cytotoxic properties of okadaic acid at similar concentrations have been reported previously, including our work on insect haemocytes (Coates et al. 2019). DSP toxins are known to promote paracellular permeability in vitro (e.g., epithelial T84 cell monolayers; Tripuraneni et al. 1997) and okadaic acid can make its way from the luminal space to the blood in vivo (e.g., rodents; Ehlers et al. 2011). Epithelial sloughing, displacement into the lumen, vacuolisation, and cell death of the intoxicated larval gut are pathological features shared among many studies conducted in rodents. For example, intragastric intubation of okadaic acid at similar doses provoked villus fragmentation, shedding of intestinal and colonic epithelial cells, lesions, apoptosis and necrosis in the liver, kidney and forestomach in Nude and Wistar rats, and CD-1 mice (Berven et al. 2001; Liu et al. 2020Vieira et al. 2018). There is, however, variation in the extent of toxin sensitivity and pathological states among rodent models/species/strains of DSP, which may extend to different sources of G. mellonella (Coates et al. 2019 and references cited therein). Nevertheless, wax moth larvae provide a convenient platform for tracking broad histopathological changes in the absence/presence of biotic/ abiotic factors as their size permits simultaneous assessment of multiple tissues/organs (Perdoni et al. 2014; Emery et al. 2019).

Most of the risk assessments available for marine toxins like okadaic acid are based on single, acute events performed on mice or in vitro using mammalian cell lines with a view to determining broad toxicological endpoints, such as, $\mathrm{LD}_{50}$, lowest-observed-adverse-effects values and toxicity equivalency factors (e.g., Abal et al. 2018). The effects of continuous exposure of humans to contaminated shellfish tissue at market acceptable quantities (i.e., $<160 \mu \mathrm{g} / \mathrm{kg}$ ) are largely uncharacterised. Some recent efforts have focussed on subacute or chronic low-dose exposure to determine longer term impacts on human health, and to a lesser extent, the shellfish hosts in situ. Liu et al. (2020) fed rats $80 \mu \mathrm{g} / \mathrm{kg}$ okadaic acid for 30 days, noting significant changes in colonic microbial loads within 3 days, in addition to local erosion of epithelial cells. Intraspecific variation in microbial abundance among intoxicated rats increased over the 30 days. Microbial diversity fluctuated, but there was a persistent inverse relationship between Firmicutes and Bacteroides abundances-signatures shared with our data. Daily intraperitoneal injections of $0.2-10 \mu \mathrm{g} / \mathrm{kg}$ for 120 days affected negatively the liver hepatocytes of mice (distorted endoplasmic reticulum, compromised mitochondrial membrane integrity, and apoptosis), as well as elevated levels of malondialdehyde (lipid peroxidation product) in serum (Wang et al. 2021). Proteomic assessment of the intoxicated mice retrieved 46 differentially expressed proteins with functions spanning metabolism, stress/chaperone factors, apoptosis, and the cytoskeleton. Although okadaic acid-induced DNA damage, modulation of DNA repair mechanisms, and links to tumour formation have been reported widely, they vary according to cell-line and/or host strain (Souid-Mensi et al. 2008; Valdiglesias et al. 2010, 2013). Long-term, pernicious effects of okadaic acid and DSP toxins are a considerable risk factor for gastrointestinal, hepatic, and pancreatic cancer according to several studies that co-correlated harmful algal bloom events over several decades with tumorigenesis along the 

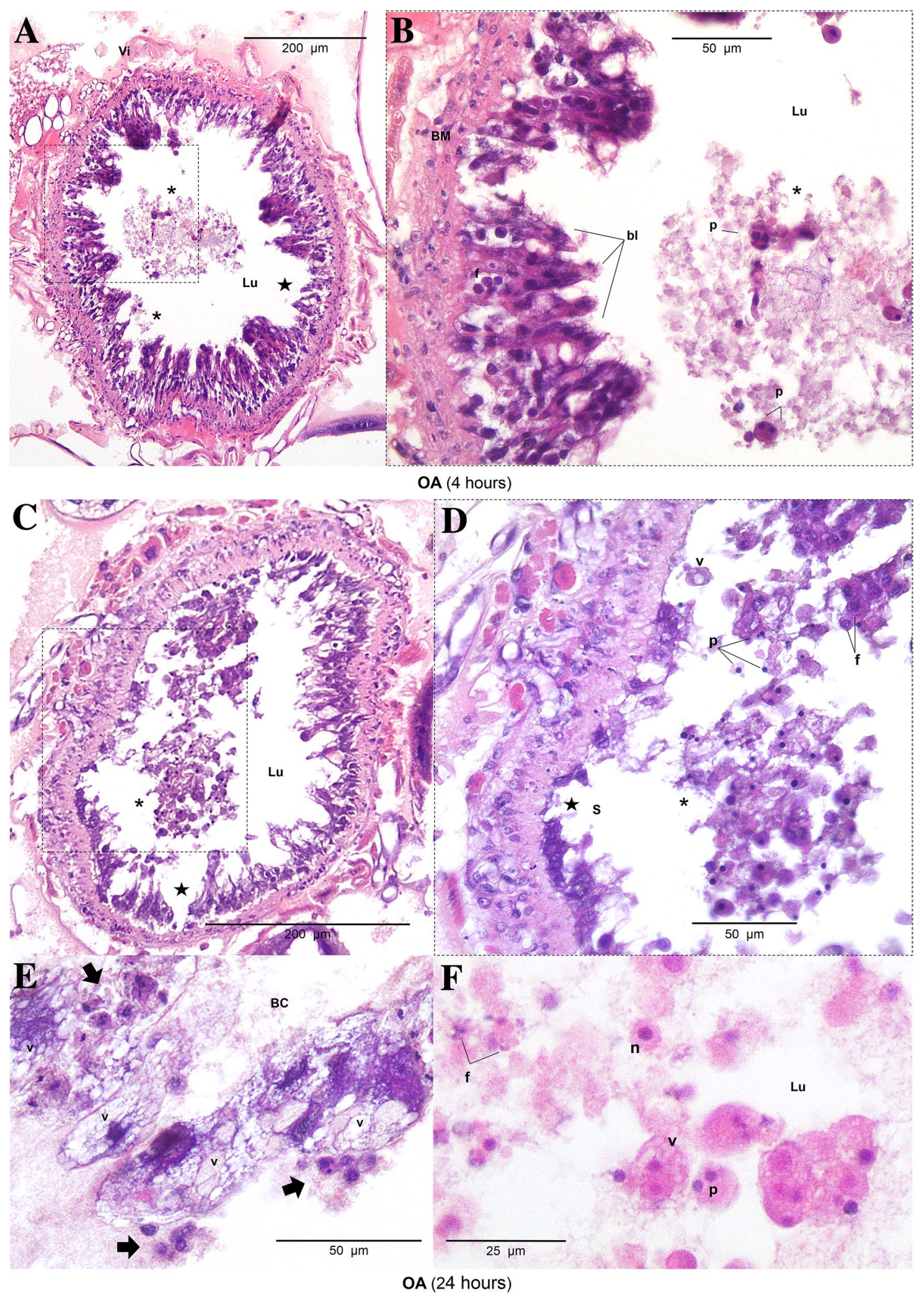

Fig. 6 Tissue sections of the Galleria mellonella midgut when forcefed okadaic acid $(240 \mu \mathrm{g} / \mathrm{kg})$. Photomicrographs depict transverse sections at 4 (panels A, B), 24 (panels C, D, E, F), and 48 (panels $\mathrm{G}, \mathrm{H})$ h post-inoculation. $B C$ body cavity; $B M$, basement membrane; $b l$, blebbing/blistering; ef epithelia fold(s); $f$, fragmentation (karyorrhexis); $k$, karyolysis; $L u$ lumen; $n$ normal nucleus; $p$ pyknosis; $v$ vacuolisation; vi visceral muscle tissue. An asterisk $(*)$ indicates cellular displacement. Black stars highlight areas where damage has completely removed epithelia and exposed underlying muscle. Black arrows point to compromised haemocytes. Panels $\mathrm{G}$ and $\mathrm{H}$ represent tissues at $48 \mathrm{~h}$ post-inoculation of okadaic acid ( $240 \mu \mathrm{g} / \mathrm{larva})$ 


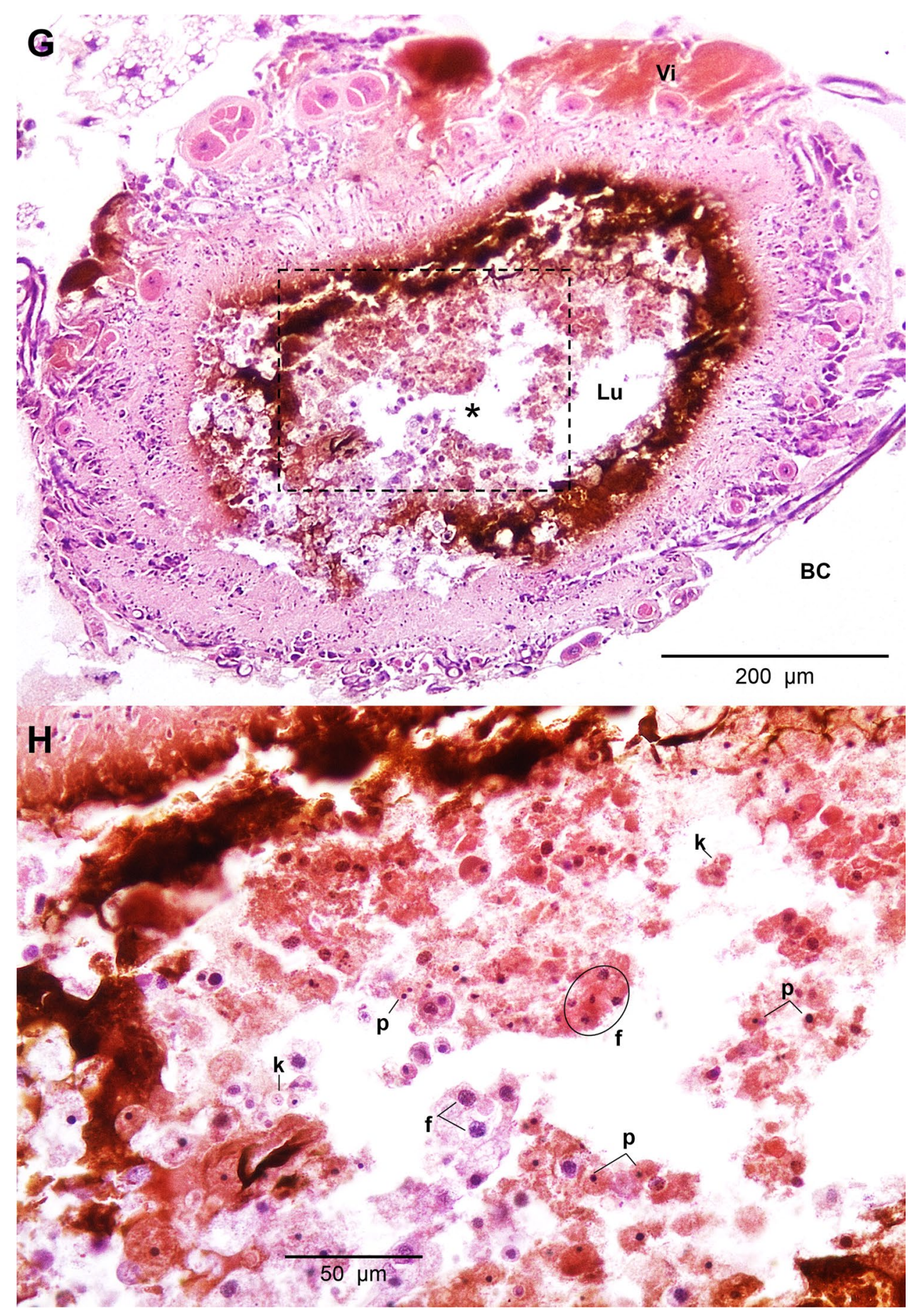

Fig. 6 (continued)

alimentary canal (Cordier et al. 2000; Lopez-Rodas et al. 2006; Manerio et al. 2008; Del Campo et al. 2013).

Despite the increasing popularity of G. mellonella for general screening of microbes, toxins, and food additives (Champion et al. 2016; Maguire et al. 2016; Pereira et al. 2020; Emery et al. 2021), most experimental approaches inoculate directly into the body cavity (intrahaemocoelic injection)-circumventing natural routes of exposure for the GI tract-and relatively few studies monitor the microbiome. There is consensus, however, that enterococci are the most abundant and prevalent bacterial resident in the gut (Fig. 8; Bucher 1963; Jarosz 1979; Johnston and Rolff 2015; Dubovskiy et al. 2016; Krams et al. 2017; Ignasiak and Maxwell 2018; Allonsius et al. 2019; Polenogova et al. 


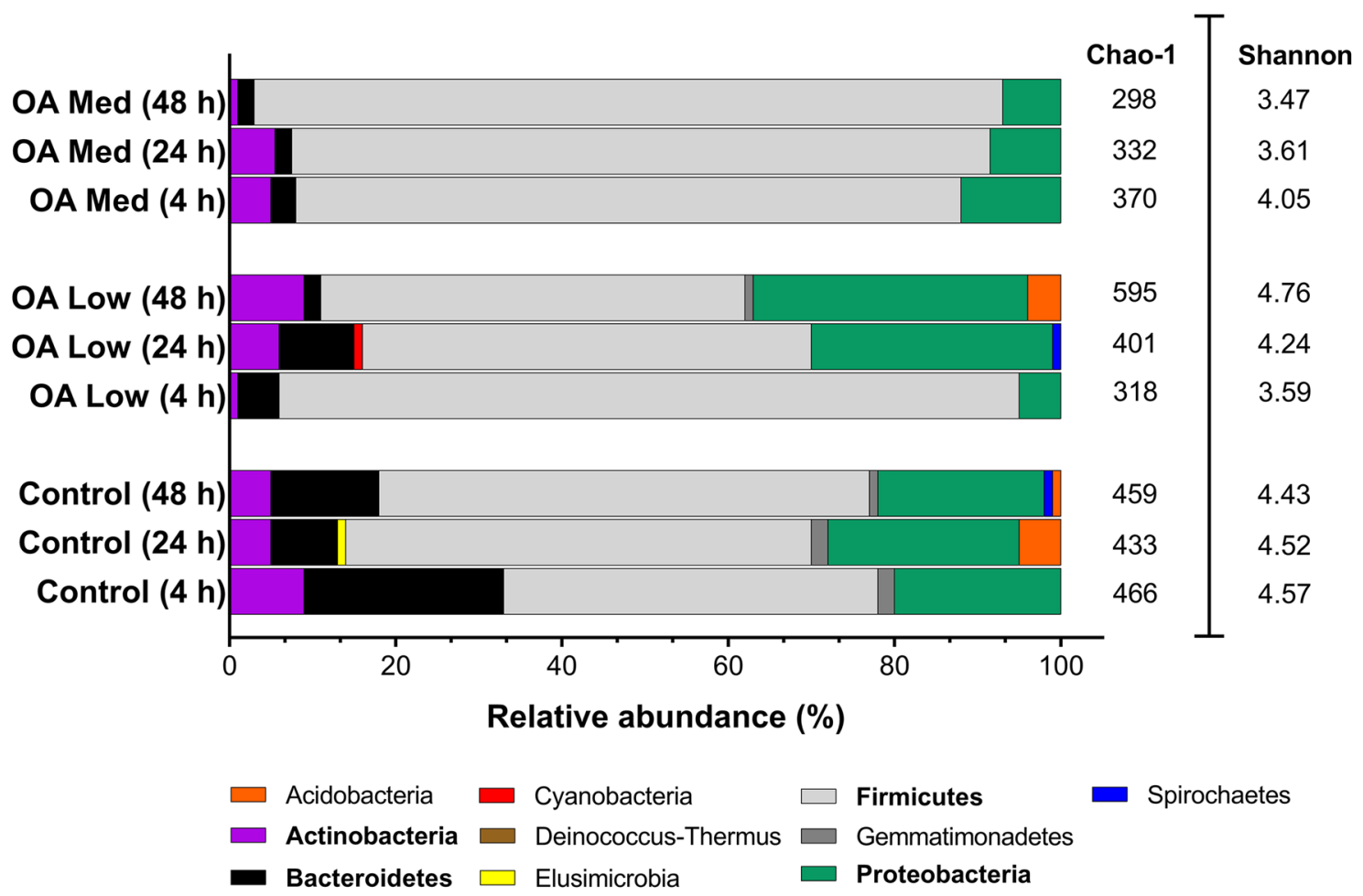

Fig. 7 Phylum-level bacterial composition (16S rRNA V3-V4) of the gut tissues of Galleria mellonella during okadaic acid $\left(\mathrm{C}_{44} \mathrm{H}_{68} \mathrm{O}_{13}\right)$ intoxication. Insects were force-fed PBS $(+5 \% \mathrm{v} / \mathrm{v}$ DMSO) or okadaic acid $(\mathrm{OA} ; 80 \mu \mathrm{g} / \mathrm{kg}$ [low], $240 \mu \mathrm{g} / \mathrm{kg}$ [med]) and incubated at $30{ }^{\circ} \mathrm{C}$ in the dark. At 4,24 , and $48 \mathrm{~h}$ post-intoxication, insects were

2019; Kryukov et al. 2021). Enterococcus species, notably E. mundtii, are considered heritable symbionts that assist in managing the microbiota during metamorphosis and disabling them through targeted antibiotic treatment can make way for pathobionts to emerge (e.g., Serratia; Bucher 1963; Johnston and Rolff 2015). The low bacterial diversity/ richness indices in G. mellonella can be attributed to their diet—based on wax and honey, which are replete in natural antimicrobials - and the bacteriocin-producing enterococci (Jarosz 1970; Johnston and Rolff 2015). Interestingly, Kong et al. (2019) demonstrated that long-chain hydrocarbon beeswax - the main dietary component of larvae-can be metabolised in the absence of microbes.

Infection of $G$. mellonella larvae via an oral route with bacteria (Bacillus thuringiensis; Dubovskiy et al. 2016), fungi (Cordyceps militaris; Kryukov et al. 2020), or parasite envenomation (Habrobracon hebetor; Polenogova et al. 2019) each promote dysbiosis - characterised by a common shift in Firmicutes relative abundances to either Proteobacteria- or Bacteroidetes-dominance, and reductions in diversity/richness indices. Again, our findings complement these reports (Fig. 7). Such dysbiotic events tend to enable pathogenic or opportunistic residents to proliferate and compromise the host-increasing their vulnerability to disease (e.g., dissected and the gut tissues were snap frozen in liquid nitrogen ( $n=9$ per treatment across time points). Inset, average Chao-1, and Shannon richness/diversity indices are placed next to the respective treatment/time point. The corresponding values for the extraction blank have been filtered out

spontaneous bacteriosis). We do observe changes in the $G$. mellonella gut microbiome and tissue architecture in the presence of okadaic acid, which can be linked to enhanced susceptibility to infection with a non-pathogen strain of $E$. coli (K12; Fig. 3). Antibiotics, such as oxytetracycline, can be used to disturb or purge gnotobiotic G. mellonella of the microbiota (Ignasiak and Maxwell 2018), and alongside their capacity to act as a host for many bacterial causes of gastroenteritis (e.g., Helicobacter pylori; Giannouli et al. 2014; Vibrio parahaemolyticus; Wagley et al. 2018, enteroaggregative Escherichia coli, Guerrieri et al. 2019), their use could extend to modelling/trialling novel antibiotic therapies against gut pathogens and scoping collateral consequences on the resident microbes and tissues.

\section{Concluding remarks}

Doses of okadaic acid exceeding $160 \mu \mathrm{g} / \mathrm{kg}$ cause obvious gut erosion, cellular distortion, displacement, and death along the alimentary canal in both rodent and insect models and are linked to dysbiosis. Whether okadaic acid influences the microbiota directly or such changes are indirect due to a decline in pathological condition (i.e., gastropathy) remains 


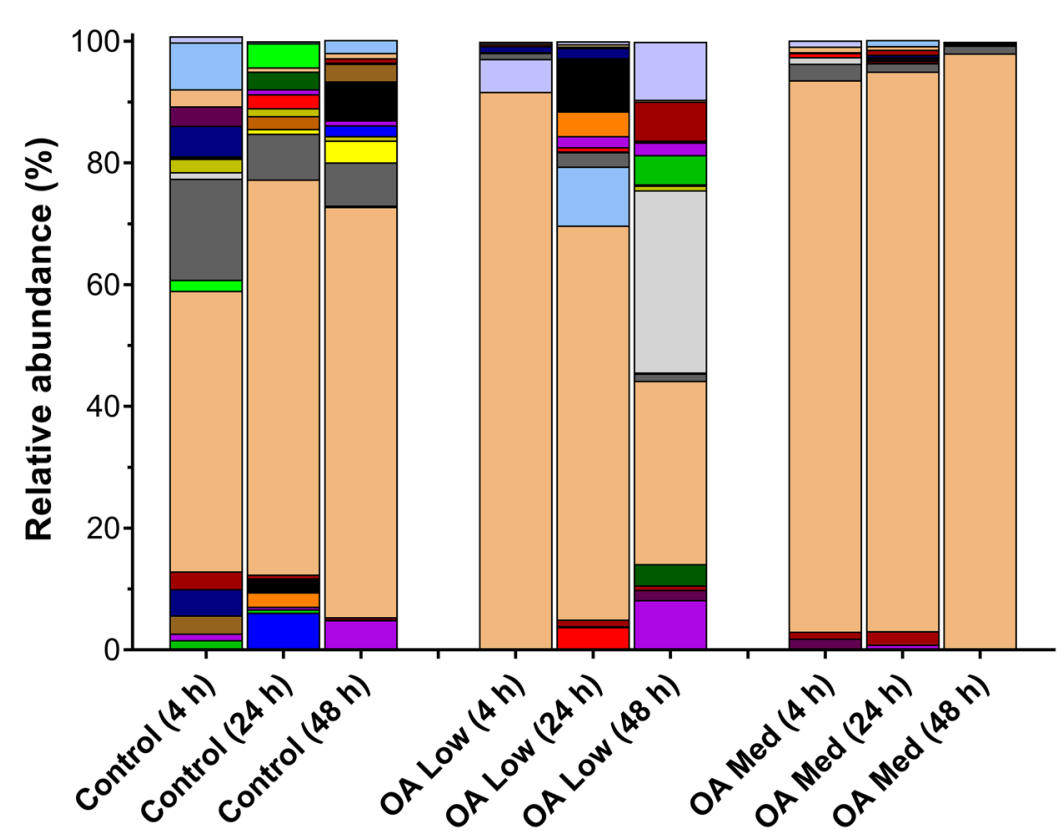

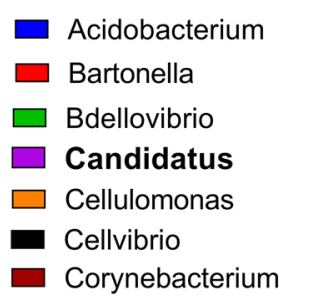

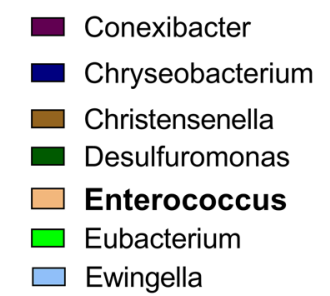

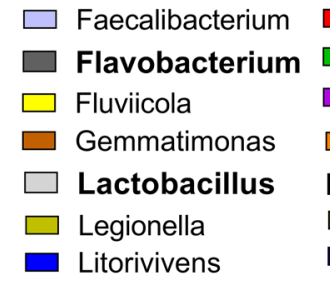

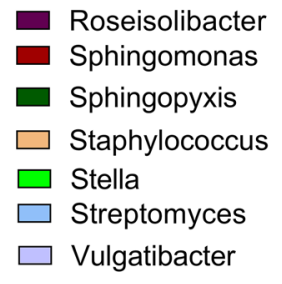

Fig. 8 Genus-level bacterial composition (16S rRNA V3-V4) of the gut tissues of Galleria mellonella during okadaic acid $\left(\mathrm{C}_{44} \mathrm{H}_{68} \mathrm{O}_{13}\right)$ intoxication. Insects were force-fed PBS (+5\% v/v DMSO) or okadaic acid $\left(80 \mu \mathrm{g} / \mathrm{kg}\right.$ [low], $240 \mu \mathrm{g} / \mathrm{kg}$ [med]) and incubated at $30{ }^{\circ} \mathrm{C}$ in the dark. At 4,24 , and $48 \mathrm{~h}$ post-intoxication, insects were dis-

uncertain. Herein, we have verified that G. mellonella larvae represent a reliable model for studying okadaic acidinduced gastrotoxicity at the whole organism, tissue, and cellular levels. More broadly, we demonstrate that a sublethal dose of okadaic acid $(80 \mu \mathrm{g} / \mathrm{kg})$ is potent enough to enhance susceptibility to infection from a routine laboratory bacterium. This suggests that humans exposed repeatedly to sub-regulatory levels of okadaic acid are at an increased risk of bacteriosis-likely from an existing resident gut symbiont or pathobiont.

Supplementary Information The online version contains supplementary material available at https://doi.org/10.1007/s00204-021-03132-x.

Acknowledgements We are grateful to Dr Jessica Bevan (née Thomas, Swansea University) for providing helpful insight into the analysis of the bacterial OUT data. We would like to thank Mrs Sophie Malkin (BlueFish Technical Officer) for assistance with histology.

Author contribution CJC conceived the study. All authors performed the experiments and gathered data. HE and CJC analysed and sected and the gut tissues were snap frozen in liquid nitrogen $(n=9$ per treatment across time points). For the control ( $48 \mathrm{~h})$, 'Candidatus' represents proportions of $C$. Trichorickettsia (0.89) and C. Monilibacter $(0.11)$. For the OA Low $(48 \mathrm{~h})$, 'Candidatus' represents proportions of C. Neoehrlichia (0.35) and C. Koribacter (0.65)

interpreted the data. CJC prepared the manuscript with input from $\mathrm{HE}$ and AFR. Resources and supervision were provided by CJC. CJC revised the text.

Funding Operations were part funded by the European Regional Development Fund through the Ireland Wales Cooperation programme, BlueFish (AFR and CJC). The European Social Fund (ESF) supported $\mathrm{HE}$ in the form of a KESS2 PhD scholarship, and was supplemented via start-up funds (College of Science, Swansea University) assigned to CJC.

\section{Declarations}

Conflict of interest The authors declare that we have no conflicts of interest.

Ethical standards College of Science (Swansea University) research ethics committee approved the use of G. mellonella for toxicology work (SU-ethics-120218/470; SU-ethics-student-280619/1777).

Open Access This article is licensed under a Creative Commons Attribution 4.0 International License, which permits use, sharing, adaptation, distribution and reproduction in any medium or format, as long 
as you give appropriate credit to the original author(s) and the source, provide a link to the Creative Commons licence, and indicate if changes were made. The images or other third party material in this article are included in the article's Creative Commons licence, unless indicated otherwise in a credit line to the material. If material is not included in the article's Creative Commons licence and your intended use is not permitted by statutory regulation or exceeds the permitted use, you will need to obtain permission directly from the copyright holder. To view a copy of this licence, visit http://creativecommons.org/licenses/by/4.0/.

\section{References}

Abal P, Louzao MC, Suzuki T, Watanabe R, Vilariño N, Carrera C et al (2018) Toxic action reevaluation of okadaic acid, dinophysistoxin- 1 and dinophysistoxin-2: toxicity equivalency factors based on the oral toxicity study. Cell Physiol Biochem 49(2):743-757

Alghoribi MF, Gibreel TM, Dodgson AR, Beatson SA, Upton M (2014) Galleria mellonella infection model demonstrates high lethality of ST69 and ST127 uropathogenic E coli. PLoS ONE 9(7):e101547

Allegra E, Titball RW, Carter J, Champion OL (2018) Galleria mellonella larvae allow the discrimination of toxic and non-toxic chemicals. Chemosphere 198:469-472

Allonsius CN, Van Beeck W, De Boeck I, Wittouck S, Lebeer S (2019) The microbiome of the invertebrate model host Galleria mellonella is dominated by Enterococcus. Anim Microbiome $1(1): 1-7$

Altschul SF, Gish W, Miller W, Myers EW, Lipman DJ (1990) Basic local alignment search tool. J Mol Biol 215(3):403-410

Aune T, Larsen S, Aasen JA, Rehmann N, Satake M, Hess P (2007) Relative toxicity of dinophysistoxin-2 (DTX-2) compared with okadaic acid, based on acute intraperitoneal toxicity in mice. Toxicon 49(1):1-7

Aune T, Espenes A, Aasen JAB, Quilliam MA, Hess P, Larsen S (2012) Study of possible combined toxic effects of azaspiracid-1 and okadaic acid in mice via the oral route. Toxicon 60(5):895-906

de Barros PP, Rossoni RD, de Camargo RF, Silva MP, de Souza CM, Jorge AOC, Junqueira JC (2019) Two sporulated Bacillus enhance immunity in Galleria mellonella protecting against Candida albicans. Microb Pathog 132:335-342

Berven G, Sætre F, Halvorsen K, Seglen PO (2001) Effects of the diarrhetic shellfish toxin, okadaic acid, on cytoskeletal elements, viability and functionality of rat liver and intestinal cells. Toxicon 39(2-3):349-362

Bialojan C, Takai A (1988) Inhibitory effect of a marine-sponge toxin, okadaic acid, on protein phosphatases specificity and kinetics. Biochem J 256(1):283-290

Bresnan E, Arévalo F, Belin C, Branco MA, Cembella AD, Clarke D et al (2021) Diversity and regional distribution of harmful algal events along the Atlantic margin of Europe. Harmful Algae 102:e101976

Browne N, Heelan M, Kavanagh K (2013) An analysis of the structural and functional similarities of insect hemocytes and mammalian phagocytes. Virulence 4(7):597-603

Bucher GE (1963) Survival of populations of Streptococcus faecalis Andrewes and Horder in gut of Galleria mellonella (Linnaeus) during metamorphosis, and transmission of bacteria to filial generation of host. J Insect Pathol 5(3):336

Caporaso JG, Kuczynski J, Stombaugh J, Bittinger K, Bushman FD, Costello EK et al (2010) QIIME allows analysis of high-throughput community sequencing data. Nat Methods 7(5):335-336

Champion OL, Wagley S, Titball RW (2016) Galleria mellonella as a model host for microbiological and toxin research. Virulence 7(7):840-845
Coates CJ, Söderhäll K (2020) The stress-immunity axis in shellfish. J Invertebr Pathol 18:e107492

Coates CJ, Lim J, Harman K, Rowley AF, Griffiths DJ, Emery H, Layton W (2019) The insect, Galleria mellonella, is a compatible model for evaluating the toxicology of okadaic acid. Cell Biol Toxicol 35(3):219-232

Cordier S, Monfort C, Miossec L, Richardson S, Belin C (2000) Ecological analysis of digestive cancer mortality related to contamination by diarrhetic shellfish poisoning toxins along the coasts of France. Environ Res 84(2):145-150

Corriere M, Baptista M, Paula JR, Repolho T, Rosa R, Costa PR, Soliño L (2020) Impaired fish swimming performance following dietary exposure to the marine phycotoxin okadaic acid. Toxicon 179:53-59

Cree BA, Spencer CM, Varrin-Doyer M, Baranzini SE, Zamvil SS (2016) Gut microbiome analysis in neuromyelitis optica reveals overabundance of Clostridium perfringens. Ann Neurol 80(3):443-447

Del Campo M, Toledo H, Lagos N (2013) Okadaic acid toxin at sublethal dose produced cell proliferation in gastric and colon epithelial cell lines. Mar Drugs 11(12):4751-4760

Dhanji-Rapkova M, O'Neill A, Maskrey BH, Coates L, Alves MT, Kelly RJ et al (2018) Variability and profiles of lipophilic toxins in bivalves from Great Britain during five and a half years of monitoring: Okadaic acid, dinophysis toxins and pectenotoxins. Harmful Algae 77:66-80

Dubovskiy IM, Grizanova EV, Whitten MM, Mukherjee K, Greig C, Alikina $\mathrm{T}$ et al (2016) Immuno-physiological adaptations confer wax moth Galleria mellonella resistance to Bacillus thuringiensis. Virulence 7(8):860-870

Ehlers A, Scholz J, These A, Hessel S, Preiss-Weigert A, Lampen A (2011) Analysis of the passage of the marine biotoxin okadaic acid through an in vitro human gut barrier. Toxicology 279(1-3):196-202

Emery H, Johnston R, Rowley AF, Coates CJ (2019) Indomethacininduced gut damage in a surrogate insect model Galleria Mellonella. Arch Toxicol 93(8):2347-2360

Emery H, Butt TM, Coates CJ (2021) Nutraceutical intervention protects against bacterial and chemical-induced gastrotoxicity in a non-mammalian model Galleria mellonella. Food Chem Toxicol 154:e112354

Eren AM, Morrison HG, Lescault PJ, Reveillaud J, Vineis JH, Sogin ML (2015) Minimum entropy decomposition: unsupervised oligotyping for sensitive partitioning of high-throughput marker gene sequences. ISME J 9(4):968-979

Giannouli M, Palatucci AT, Rubino V, Ruggiero G, Romano M, Triassi M et al (2014) Use of larvae of the wax moth Galleria mellonella as an in vivo model to study the virulence of Helicobacter pylori. BMC Microbiol 14(1):1-10

Grizanova EV, Coates CJ, Dubovskiy IM, Butt TM (2019) Metarhizium brunneum infection dynamics differ at the cuticle interface of susceptible and tolerant morphs of Galleria mellonella. Virulence 10(1):999-1012

Grizanova EV, Coates CJ, Butt TM, Dubovskiy IM (2021) RNAimediated suppression of insect metalloprotease inhibitor (IMPI) enhances Galleria mellonella susceptibility to fungal infection. Dev Comp Immunol 122:e104126

Guerrieri CG, Pereira MF, Galdino ACM, Santos ALSD, Elias WP, Schuenck RP, Spano LC (2019) Typical and atypical enteroaggregative Escherichia coli are both virulent in the Galleria mellonella model. Front Micrbiol 10:e1791

Ignasiak K, Maxwell A (2018) Oxytetracycline reduces the diversity of tetracycline-resistance genes in the Galleria mellonella gut microbiome. BMC Microbiol 18(1):1-8

Jarosz J (1979) Gut flora of Galleria mellonella suppressing ingested bacteria. J Invertebr Pathol 34(2):192-198 
Jiménez-Cárcamo D, García C, Contreras HR (2020) Toxins of okadaic acid-group increase malignant properties in cells of colon cancer. Toxins 12(3):179

Johnston PR, Rolff J (2015) Host and symbiont jointly control gut microbiota during complete metamorphosis. PLoS Pathog 11(11):e1005246

Kisand V, Cuadros R, Wikner J (2002) Phylogeny of culturable estuarine bacteria catabolizing riverine organic matter in the northern Baltic Sea. App Environ Microbiol 68(1):379-388

Kong HG, Kim HH, Chung JH, Jun J, Lee S, Kim HM et al (2019) The Galleria mellonella hologenome supports microbiota-independent metabolism of long-chain hydrocarbon beeswax. Cell Rep 26(9):2451-2464

Krachler AM, Sirisaengtaksin N, Monteith P, Paine CT, Coates CJ, Lim J (2021) Defective phagocyte association during infection of Galleria mellonella with Yersinia pseudotuberculosis is detrimental to both insect host and microbe. Virulence 12(1):638-653

Krams IA, Kecko S, Jõers P, Trakimas G, Elferts D, Krams R et al (2017) Microbiome symbionts and diet diversity incur costs on the immune system of insect larvae. J Exp Biol 220(22):4204-4212

Kryukov VY, Kosman E, Tomilova O, Polenogova O, Rotskaya U, Tyurin M (2020) Interplay between fungal infection and bacterial associates in the wax moth Galleria mellonella under different temperature conditions. J Fungi 6(3):170

Lange A, Schäfer A, Bender A, Steimle A, Beier S, Parusel R, Frick JS (2018) Galleria mellonella: a novel invertebrate model to distinguish intestinal symbionts from pathobionts. Front Immunol 9:2114

Lange A, Schäfer A, Frick JS (2019) A Galleria mellonella oral administration model to study commensal-Induced innate immune responses. JoVE 145.

Lim J, Coates CJ, Seoane PI, Garelnabi M, Taylor-Smith LM, Monteith P et al (2018) Characterizing the mechanisms of nonopsonic uptake of cryptococci by macrophages. J Immunol 200(10):3539-3546

Liu Y, Zheng JW, Peng XC, Li HY, Huang L, Li DW et al (2020) Changes in colonic microbiotas in rat after long-term exposure to low dose of okadaic acid. Chemosphere 254:e126874

Loh JM, Adenwalla N, Wiles S, Proft T (2013) Galleria mellonella larvae as an infection model for group A streptococcus. Virulence 4(5):419-428

Lopez-Rodas V, Maneiro E, Martínez J, Navarro M, Costas E (2006) Harmful algal blooms, red tides and human health: Diarrhetic shellfish poisoning and colorectal cancer. An R Acad Nac Farm 72(3):391-408

Louzao MC, Fernández DA, Abal P, Fraga M, Vilariño N, Vieytes MR, Botana LM (2015) Diarrhetic effect of okadaic acid could be related with its neuronal action: Changes in neuropeptide Y. Toxicol Lett 237(2):151-160

Louzao MC, Costas C, Abal P, Suzuki T, Watanabe R, Vilariño N et al (2021) Serotonin involvement in okadaic acid-induced diarrhoea in vivo. Arch Toxicol 95:2797-2813

Magoč T, Salzberg SL (2011) FLASH: fast length adjustment of short reads to improve genome assemblies. Bioinformatics 27(21):2957-2963

Maguire R, Duggan O, Kavanagh K (2016) Evaluation of Galleria mellonella larvae as an in vivo model for assessing the relative toxicity of food preservative agents. Cell Biol Toxicol 32(3):209-216

Manerio E, Rodas VL, Costas E, Hernandez JM (2008) Shellfish consumption: a major risk factor for colorectal cancer. Med Hypotheses 70(2):409-412

Matias WG, Creppy EE (1996) Transplacental passage of [3H]-okadaic acid in pregnant mice measured by radioactivity and high-performance liquid chromatography. Hum Exp Toxicol 15(3):226-230
McCarron P, Kilcoyne J, Hess P (2008) Effects of cooking and heat treatment on concentration and tissue distribution of okadaic acid and dinophysistoxin-2 in mussels (Mytilus edulis). Toxicon 51(6):1081-1089

Moya-Andérico L, Vukomanovic M, del Mar CM, Segura-Feliu M, Gil V, José A, Torrents E (2021) Utility of Galleria mellonella larvae for evaluating nanoparticle toxicology. Chemosphere 266:e129235

Munday R (2013) Is protein phosphatase inhibition responsible for the toxic effects of okadaic acid in animals? Toxins 5(2):267-285

O'Mahony M (2018) EU regulatory risk management of marine biotoxins in the marine bivalve mollusc food-chain. Toxins 10(3):118

Perdoni F, Falleni M, Tosi D, Cirasola D, Romagnoli S, Braidotti P, Borghi E (2014) A histological procedure to study fungal infection in the wax moth Galleria mellonella. Eur J Histochem 58(3):2428

Pereira MF, Rossi CC, da Silva GC, Rosa JN, Bazzolli DMS (2020) Galleria mellonella as an infection model: an in-depth look at why it works and practical considerations for successful application. Pathog Dis 78(8):ftaa056

Piatek M, Sheehan G, Kavanagh K (2020) Utilising Galleria mellonella larvae for studying in vivo activity of conventional and novel antimicrobial agents. Pathog Dis 78(8):ftaa59

Polenogova OV, Kabilov MR, Tyurin MV, Rotskaya UN, Krivopalov AV, Morozova VV et al (2019) Parasitoid envenomation alters the Galleria mellonella midgut microbiota and immunity, thereby promoting fungal infection. Sci Rep 9(1):1-12

Reboreda A, Lago J, Chapela MJ, Vieites JM, Botana LM, Alfonso A, Cabado AG (2010) Decrease of marine toxin content in bivalves by industrial processes. Toxicon 55(2-3):235-243

Reguera B, Riobó P, Rodríguez F, Díaz PA, Pizarro G, Paz B et al (2014) Dinophysis toxins: causative organisms, distribution and fate in shellfish. Mar Drugs 12(1):394-461

Schloss PD, Gevers D, Westcott SL (2011) Reducing the effects of PCR amplification and sequencing artifacts on 16S rRNA-based studies. PLoS ONE 6(12):e27310

Souid-Mensi G, Moukha S, Mobio TA, Maaroufi K, Creppy EE (2008) The cytotoxicity and genotoxicity of okadaic acid are cell-line dependent. Toxicon 51(8):1338-1344

Tachibana K, Scheuer PJ, Tsukitani Y, Kikuchi H, Van Engen D, Clardy J et al (1981) Okadaic acid, a cytotoxic polyether from two marine sponges of the genus Halichondria. J Am Chem Soc 103(9):2469-2471

Trainer VL, Moore L, Bill BD, Adams NG, Harrington N, Borchert J et al (2013) Diarrhetic shellfish toxins and other lipophilic toxins of human health concern in Washington State. Mar Drugs 11(6): 1815-1835

Tripuraneni J, Koutsouris A, Pestic L, De Lanerolle P, Hecht G (1997) The toxin of diarrheic shellfish poisoning, okadaic acid, increases intestinal epithelial paracellular permeability. Gastroenterology 112(1):100-108

Turner PKM, Miao VP, Palmer JD (1999) Investigating deep phylogenetic relationships among cyanobacteria and plastids by small subunit rRNA sequence analysis 1 . J Eukaryot Microbiol 46(4):327-338

Turner AD, Lewis AM, Bradley K, Maskrey BH (2021) Marine invertebrate interactions with harmful algal blooms-implications for one health. J Invertebr Pathol e107555.

Valdiglesias V, Méndez J, Pásaro E, Cemeli E, Anderson D, Laffon B (2010) Assessment of okadaic acid effects on cytotoxicity, DNA damage and DNA repair in human cells. Mutat Res Fundam Mol Mech Mutagen 689(1-2):74-79

Valdiglesias V, Prego-Faraldo MV, Pásaro E, Méndez J, Laffon B (2013) Okadaic acid: more than a diarrheic toxin. Mar Drugs 11(11):4328-4349

Vieira AC, Rubiolo JA, López-Alonso H, Cifuentes JM, Alfonso A, Bermúdez R et al (2013) Oral toxicity of okadaic acid in mice: 
Study of lethality, organ damage, distribution and effects on detoxifying gene expression. Toxins 5(11):2093-2108

Vilariño N, Louzao MC, Abal P, Cagide E, Carrera C, Vieytes MR, Botana LM (2018) Human poisoning from marine toxins: Unknowns for optimal consumer protection. Toxins 10(8):324

Wagley S, Borne R, Harrison J, Baker-Austin C, Ottaviani D, Leoni F et al (2018) Galleria mellonella as an infection model to investigate virulence of Vibrio parahaemolyticus. Virulence 9(1):197-207

Wang J, Lin L, Wang DZ (2021) Quantitative proteomic analysis reveals novel insights into hepatic toxicity in mice exposed chronically to okadaic acid. Sci Total Environ 775:145772
Whitten MMA, Coates CJ (2017) Re-evaluation of insect melanogenesis research: views from the dark side. Pigm Cell Mel Res 30(4):386-401

Yasumoto T, Murata M, Oshima Y, Sano M, Matsumoto GK, Clardy J (1985) Diarrhetic shellfish toxins. Tetrahedron 41(6):1019-1025

Publisher's Note Springer Nature remains neutral with regard to jurisdictional claims in published maps and institutional affiliations. 